\title{
Medium resolution magneto-optical Faraday rotation measurement system ${ }^{\dagger}$
}

\author{
SANDIP DHARA, V N MOORTHY, A C RASTOGI and B K DAS \\ Materials Division, National Physical Laboratory, New Delhi 110012, India
}

\begin{abstract}
Medium resolution magneto-optic Faraday rotation measurement setup has been reported. Measuremental setup reported is made up of simple polarized laser source. beam expander, analyzer and a bisected cross polarized $p-n$ detector for single ended measurements. Result of $\mathrm{CoO}$ modified yttrium iron garnet (YIG) films showed a rotation of $3 \mathrm{deg} / \mu \mathrm{m}$ which is comparable to earlier reported Co-doped YIG films.
\end{abstract}

Keywords. Magneto-optic; Faraday rotation; Kerr rotation; $\mathrm{He}-\mathrm{Ne}$ laser; polarization; perpendicular magnetization; photo detector.

\section{Introduction}

Optically active magnetic materials have been extensively investigated for their application in memory and computational devices (Abe and Gomi 1987a). Thin film media has been the main object of attention for such purposes. An important criterion of these materials for application in recording purpose is their easy axis of magnetization lying perpendicular to the film plane. Materials having perpendicular easy axis have been observed to exhibit large magneto-optical (MO) effect (Argyres 1955). To measure the MO effect of these materials, two modes are used: (i) the reflection mode (Kerr effect) which is observed in the metallic thin films, and (ii) the transmission mode (Faraday effect) mainly observed in the oxide thin films. Oxide films, because of their well-known advantages over the metallic films have been the main topic of research. Because the oxide films transmit light, the Faraday effect is used instead of the Kerr effect in the signal retrieval in the magneto-optical recording media. Faraday rotation $\left(\theta_{F}\right)$ is the angle of rotation of polarization of an incident light beam when it is passed through a medium magnetized or having a component of magnetization along the direction of incidence. Amount of rotation $\left(\theta_{F}\right)$ is a function of the value of magnetization lying along the direction of incidence and the thickness of the medium. Magneto-optic Faraday rotation measurement can be done by three different techniques (Tognetti and Borsa 1988). In the first technique linearly polarized light is sent through a magnetized material with perpendicular easy axis and the outcoming light polarization plane is determined by finding the analyzer setting minimizes the light intensity reaching the detector. Resolution of the measurement of $\theta_{\mathrm{F}}$ is certainly poor as it will be restricted solely by the least count of rotation of analyzer assembly. In the second method, $\theta_{\mathbf{F}}$ can be measured with better resolution than the earlier technique using polarization modulator. In this method linearly polarized light is activated alternatively in the +1 or -1 state by using electro-optic devices. Without external field, the lock-in-amplifier (LIA) reads minimum for analyzer setting $\theta=0$ and with the application of the field, the LIA reads zero with $\theta=\theta_{\mathrm{F}}$. In the third technique, $\theta_{\mathrm{F}}$ can be measured with high resolution

\footnotetext{
${ }^{\dagger}$ Paper presented at the poster session of MRSI AGM VI, Kharagpur, 1995
} 


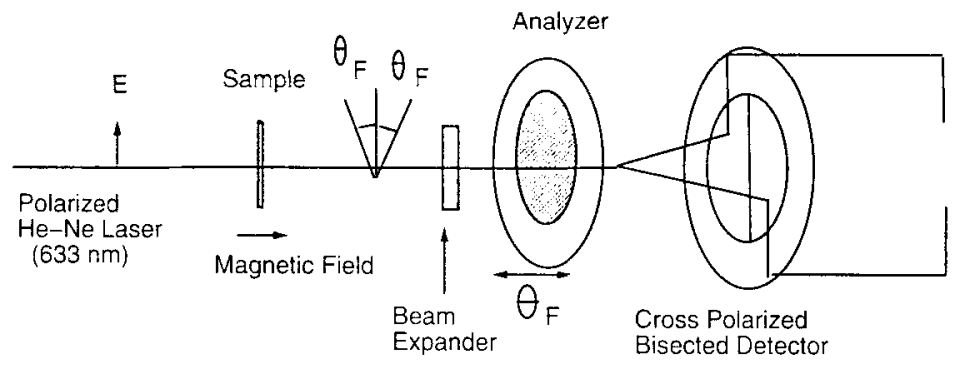

Figure 1. Schematic of magneto-optic setup.

using intensity modulation and polarizing beam splitter. In this method LIA is used in differential mode to read minimum for analyzer setting of $\theta=0$ and $\theta=\theta_{\mathrm{F}}$, respectively, without and with magnetization.

In this paper we describe a setup for measuring the Faraday rotation angle in the magnetic garnet (oxide) thin films by using the principle of first of the above mentioned techniques. Instead of the single ended output as used in the first technique (Tognetti and Borsa 1988), we have used a bisected detector to analyze the output signal in differential mode. We also present the results of the measurements performed in the Co-substituted garnet films along with the calibration parameters.

\section{Experimental}

A magneto-optical (MO) Faraday tracer comprised of a polarized $\mathrm{He}-\mathrm{Ne}$ laser $(633 \mathrm{~nm})$, a telescopic beam expander, an analyzer and a cross-polarized bisected $p-n$ detector was fabricated for measurements in the transmission mode (figure 1). The detector configuration was such that any change in the incident polarization angle would increase the output signal for one half of the detector as it was covered with a parallel polarizer and decrease the output in the other half as it was covered with a cross-polarizer, making the differential output double for an unit change in the incident polarization angle. The detector designed in the above configuration has increased resolution of measurement of $\theta_{\mathrm{F}}$. Since any rotation in polarization axis will add the intensity of $+\delta I$ in one half and $-\delta I$ in another half of the detector with a net difference of $2 \delta I$, so for a $\delta I$ resolution of the detector $\theta_{\mathrm{F}(\min )}$ will be halved, i.e. the angular resolution will be doubled.

Linearly polarized $\mathrm{He}-\mathrm{Ne}$ laser light $(633 \mathrm{~nm})$ was sent through an analyzer placed at $45^{\circ}$ alignment with the polarizer via a beam expander. The outcoming light polarization plane was determined by reading the change in the detector outputs set in the differential mode. Initially without magnetization (sample placed before beam expander and analyzer) magnetization differential output was set to minimum by adjusting the cross-polarized bisected detector. Now with magnetization on, a change in polarization angle of incident beam would cause a non-zero differential output. Analyzer being set at $45^{\circ}$ with respect to polarizer will have maximum sensitivity to the change in polarization angle of the incident polarized beam, as explained in figure 2 . The analyzer can be rotated by a spring loaded micrometer. The spring was set at a distance of $\approx 7 \mathrm{~cm}$ from the centre of the analyzer disc. For a small 


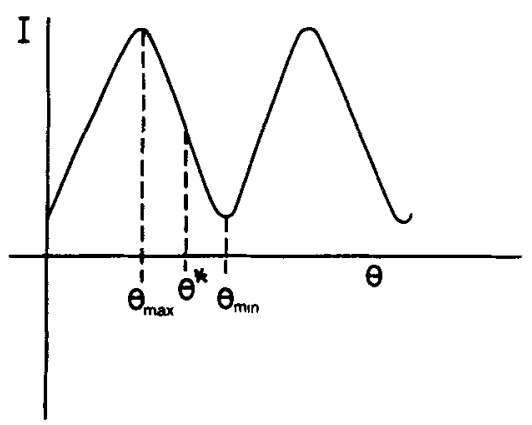

$$
\begin{aligned}
& 1(\theta)=\operatorname{los}_{0} \operatorname{Cos}^{2}\left(\theta+A_{0}\right)+\operatorname{Const} \\
& \frac{\Delta I}{\Delta \theta}=I_{11} 2 \operatorname{Sin} 2\left(\theta+H_{.,}\right) \\
& \text {AT }\left(\theta+\theta_{0}\right)=\mathrm{n} \frac{\pi}{2} \quad(\mathrm{n}=\text { Odil integer }) \\
& \frac{\Delta \mathrm{I}}{\Delta \theta}=0 \quad(\mathrm{POOR} \text { SE } I \text { SITIVITY }) \\
& \text { AT }\left(\theta+\theta_{0}\right)=\mathrm{n} \frac{\pi}{4} \quad(\mathrm{n}=\text { Odd integer }) \\
& \frac{\Delta \mathrm{I}}{\Delta \theta}=2 \mathrm{I}_{\circ} \text { (MANMUM SENSITIIT) }
\end{aligned}
$$

Figure 2. Details of analyzer setting for maximum sensitivity.

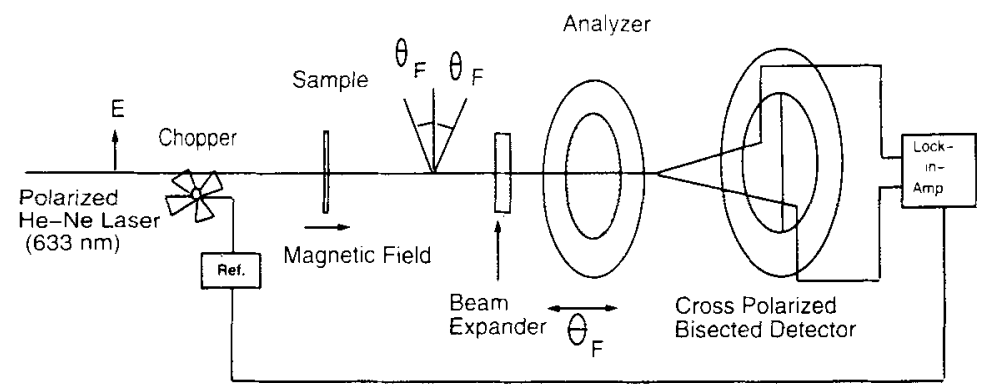

Figure 3. Schematic of intensity modulated magneto-optic setup.

rotation we can consider

$$
s=r \theta
$$

where $\theta$ is in radian.

Thus one full turn of micrometer which corresponds to a translational motion of $0.05 \mathrm{~cm}(\mathrm{~s})$ for which $\theta$ value can be calculated to be $0.2 \mathrm{deg}$. The detector output has a minimum resolution of $0.5 \mathrm{mV}$ which corresponds to $0.2 \mathrm{deg}$ of rotation in the polarization angle. Thus a Faraday tracer was developed with a medium resolution of $0.2 \mathrm{deg}$. The system requires further development where LIA assisted intensity modulated technique (figure 3) will be adapted for high resolution measurements (Dhara et al 1995) following the principle of the third technique described earlier.

\section{Results and discussions}

Recently we have developed $\mathrm{CoO}$ adsorbed yttrium iron garnet (YIG) films (CoO/YIG) for introducing perpendicular anisotropy in the garnet media which show mostly inplane magnetization. $\mathrm{CoO} / \mathrm{YIG}$ films showed enhanced coercivity value and squareness ratio of $270 \mathrm{kA} / \mathrm{m}$ and 0.60 when measured perpendicular to the plane of the film (Moorthy et al 1995). We have measured the $\theta_{\mathrm{F}}$ of $3 \mathrm{deg} / \mu \mathrm{m}$ (film thickness $\approx 3000 \AA$ ) in our setup at a field of $80 \mathrm{kA} / \mathrm{m}$. These values are quite in agreement with the earlier results of Co-doped garnet films $\left(\mathrm{YBi}_{2} \mathrm{Fe}_{5-2 x} \mathrm{Co}_{x} \mathrm{Ge}_{x} \mathrm{O}_{12}\right)$ (Abe and Gomi 1987b). 


\section{Acknowledgements}

The authors are thankful to Mr Mukul Sharma for his technical assistance in setting up the magneto-optic system. One of the authors (SD) acknowledge the Council of Scientific and Industrial Research (CSIR), New Delhi for providing the research fellowship.

\section{References}

Abe M and Gomi M 1987a in Adv, in magneto-optics(eds) K Tushima and K Shinagawa (Tokyo: OHMSHA L.t. and North-Holland Pub. Co.) p. 299

Abe M and Gomi M 1987b J. Magn. Soc. Jpn 11299

Argyres P N 1955 Phys. Rev. 87334

Dhara S, Moorthy V N, Rastogi A C, Sharma M and Das B K 1995 (To be communicated)

Moorthy V N, Dhara S, Rastogi A C and Das B K 1995 (To be communicated)

Tognetti V and Borsa T (eds) 1988 in Magnetic properties of matter (Singapore: World Scientific Pub. Co. Pvt. Ltd) p. 363 